\title{
INTEGRAL INVOLVING ALEPH-FUNCTION AND THE GENERALIZED INCOMPLETE HYPERGEOMETRIC FUNCTION
}

\author{
D. KUMAR ${ }^{1}$, F.Y. AYANT ${ }^{2}$, F. UÇAR ${ }^{3}, \S$
}

\begin{abstract}
The aim of this paper is to establish a general definite integrals involving product of the Aleph function and generalized incomplete hypergeometric function with general arguments. Being unified and general in nature, this integral yield a number of known and new results as special cases. For the sake of illustration, several corollaries are also recorded here as special case of our main results.
\end{abstract}

Keywords: Aleph-function, $I$-function, $H$-function, generalized incomplete hypergeometric function.

AMS Subject Classification: Primary 33C20, 33C45, 33C60, 33C99; Secondary 33B20

\section{Introduction and Preliminaries}

Fractional calculus is one of the generalizations of classical calculus and it has been used successfully in various fields of science and engineering. Many earlier works on the subject of fractional calculus contain interesting description of the theory and applications of fractional calculus operators in a number of areas of mathematical analysis and mathematical physics, for example ordinary and partial differential equations, mathematical modeling, integral equations, summation of series, etc. Special functions of fractional calculus constitute a old branch of mathematics; the origins of their unified and rather complete theory date to the nineteenth century. From the point of view of the applied scientists and engineers dealing with the practical application of differential equations, the role of special functions as an important tool of mathematical analysis.

The $\aleph$-function was introduced by Südland et al. [1,2], however the notation and complete definition is presented here in the following manner in terms of the Mellin-Barnes type integral (see also, $[3,4,5,6,7,8,9,10])$ :

$$
\aleph[z]=\aleph_{p_{i}, q_{i}, c_{i} ; r}^{m, n}\left[z \mid \begin{array}{l}
\left(a_{j}, A_{j}\right)_{1, n}, \ldots,\left[c_{j}\left(a_{j}, A_{j}\right)\right]_{n+1, p_{i} ; r} \\
\left(b_{j}, B_{j}\right)_{1, m}, \ldots,\left[c_{j}\left(b_{j}, B_{j}\right)\right]_{m+1, q_{i} ; r}
\end{array}\right]:=\frac{1}{2 \pi \omega} \int_{\mathfrak{L}} \Omega_{p_{i}, q_{i}, c_{i} ; r}^{m, n}(\xi) z^{-\xi} \mathrm{d} \xi,
$$

\footnotetext{
${ }^{1}$ Department of Applied Sciences, College of Agriculture, Sumerpur-Pali, Agriculture University of Jodhpur, 342304, India.

e-mail: dinesh_dino03@yahoo.com; ORCID: https://orcid.org/0000-0001-5415-1777.

${ }^{2}$ Collége Jean L'herminier, Allée des Nymphéas, 83500 La Seyne-sur-Mer, France.

e-mail: fredericayant@gmail.com; ORCID: https://orcid.org/0000-0003-0651-294X.

${ }^{3}$ Department of Mathematics, Faculty of Science and Letters, Marmara University, TR-34722 Kadiköy, Istanbul, Turkey.

e-mail: fucar@marmara.edu.tr; ORCID: https://orcid.org/0000-0002-9180-3102.

$\S$ Manuscript received: November 4, 2018; accepted: September 3, 2019.

TWMS Journal of Applied and Engineering Mathematics, Vol.10, No.3 (C) Işık University, Department of Mathematics, 2020; all rights reserved.
} 
for all $z \in \mathbb{C}, z \neq 0$, where $\omega=\sqrt{-1}$ and

$$
\Omega_{p_{i}, q_{i}, c_{i} ; r}^{m, n}(\xi)=\frac{\prod_{j=1}^{m} \Gamma\left(b_{j}+B_{j} \xi\right) \prod_{j=1}^{n} \Gamma\left(1-a_{j}-A_{j} \xi\right)}{\sum_{i=1}^{r} c_{i}\left\{\prod_{j=m+1}^{q_{i}} \Gamma\left(1-b_{j i}-B_{j i} \xi\right) \prod_{j=n+1}^{p_{i}} \Gamma\left(a_{j i}+A_{j i} \xi\right)\right\}}
$$

The integration path $\mathfrak{L}=\mathfrak{L}_{\omega \gamma \infty}(\gamma \in \mathbb{R})$ ranging from $\gamma-\omega \infty$ to $\gamma+\omega \infty$ is a contour of the Mellin-Barnes type integral, and is such that the poles of $\Gamma\left(1-a_{j}-A_{j} \xi\right), j=(\overline{1, n})$ (the symbol $(\overline{1, n})$ is used for $1,2, \ldots, \mathrm{n})$ do not coincide with the poles of $\Gamma\left(b_{j}+B_{j} \xi\right), j=$ $(\overline{1, m})$. The empty product in (2) is interpreted as unity. The parameters $p_{i}, q_{i} \in \mathbb{N}_{0}$ with $0 \leq n \leq p_{i}, 1 \leq m \leq q_{i}, c_{i}>0$ for $i=(\overline{1, r})$. The parameters $A_{j}, B_{j}, A_{j i}, B_{j i}>$ 0 and $a_{j}, b_{j}, a_{j i}, b_{j i} \in \mathcal{C}$. The existence of the Aleph-function defined on (1) depends on the following conditions:

$$
\begin{gathered}
\varphi_{l}>0, \quad|\arg (z)|<\frac{\pi}{2} \varphi_{l}, \quad l=\overline{1, r} \\
\varphi_{l} \geq 0, \quad|\arg (z)|<\frac{\pi}{2} \varphi_{l} \text { and } \Re\left\{\zeta_{l}\right\}+1<0,
\end{gathered}
$$

where

$$
\begin{gathered}
\varphi_{l}=\sum_{j=1}^{n} A_{j}+\sum_{j=1}^{m} B_{j}-c_{l}\left(\sum_{j=n+1}^{p_{l}} A_{j l}+\sum_{j=m+1}^{q_{l}} B_{j l}\right), \\
\zeta_{l}=\sum_{j=1}^{m} b_{j}-\sum_{j=1}^{n} a_{j}+c_{l}\left(\sum_{j=m+1}^{q_{l}} b_{j l}-\sum_{j=n+1}^{p_{l}} a_{j l}\right)+\frac{1}{2}\left(p_{l}-q_{l}\right), \quad l=\overline{1, r} .
\end{gathered}
$$

The Aleph function is a new special function which is an extension of the $I$-function, which itself is a generalization of the well-known and familiar $G$ - and $H$-functions in one variable. A special case of the Aleph function arises naturally in the study of fractional driftless Fokker-Planck equations with power law diffusion coefficients.

Remark 1.1. Take $c_{i}=1 \quad(i=\overline{1, r})$ in (1), yields the I-function due to Saxena [11] defined in the following manner:

$$
\begin{gathered}
I_{p_{i}, q_{i} ; r}^{m, n}(z)=\aleph_{p_{i}, q_{i}, 1 ; r}^{m, n}(z)=\aleph_{p_{i}, q_{i}, 1 ; r}^{m, n}\left[z \mid \begin{array}{c}
\left(a_{j}, A_{j}\right)_{1, n},\left[1\left(a_{j i}, A_{j i}\right)\right]_{n+1, p_{i} ; r} \\
\left(b_{j}, B_{j}\right)_{1, m},\left[1\left(b_{j i}, B_{j i}\right)\right]_{m+1, q_{i} ; r}
\end{array}\right] \\
=\frac{1}{2 \pi \omega} \int_{\mathfrak{L}} \Omega_{p_{i}, q_{i}, 1 ; r}^{m, n}(\xi) z^{-\xi} \mathrm{d} \xi
\end{gathered}
$$

where the kernel $\Omega_{p_{i}, q_{i}, 1 ; r}^{m, n}(s)$ is given in (2). The existence conditions for the integral (7) are the same in $(3)-(6)$ with $c_{i}=1 \quad(i=\overline{1, r})$.

Remark 1.2. Further, if we set $r=1$ in (7), then (1) reduces to $H$-function defined by Fox [12] (see also, [13, 14]).

$$
H_{p, q}^{m, n}(z)=\aleph_{p_{i}, q_{i}, 1 ; 1}^{m, n}(z)=H_{p, q}^{m, n}\left[z \mid \begin{array}{c}
\left(a_{j}, A_{j}\right)_{1, p} \\
\left(b_{j}, B_{j}\right)_{1, q}
\end{array}\right]=\frac{1}{2 \pi \omega} \int_{\mathfrak{L}} \Omega_{p_{i}, q_{i}, 1 ; 1}^{m, n}(\xi) z^{-\xi} \mathrm{d} \xi,
$$

where the kernel $\Omega_{p_{i}, q_{i}, 1 ; r}^{m, n}(s)$ is given in (2). The existence conditions for the integral in (8) are the same in (7) with $r=1$. 
The generalized incomplete hypergeometric function introduced by Srivastava et al. [15, p. 675, Eqn. (4.1) and Eqn. (4.2)] is represented in the following manner:

$$
{ }_{\mathbf{p}} \gamma_{\mathbf{q}}\left[\begin{array}{c}
\left(e_{1} ; \sigma\right),\left(e_{2}\right), \cdots,\left(e_{\mathbf{p}}\right) \\
\cdots \\
\left(f_{1}\right), \cdots,\left(f_{\mathbf{q}}\right)
\end{array} \mid z\right]=\sum_{\mathbf{n}=0}^{\infty} \frac{\left(e_{1} ; \sigma\right)_{\mathbf{n}}\left(e_{2}\right)_{\mathbf{n}} \cdots\left(e_{\mathbf{p}}\right)_{\mathbf{n}}}{\left(f_{1}\right)_{\mathbf{n}} \cdots\left(f_{\mathbf{q}}\right)_{\mathbf{n}}} \frac{z^{\mathbf{n}}}{\mathbf{n} !},
$$

where the incomplete Pochhammer symbols is defined as follows:

$$
(\lambda ; \sigma)_{\mathbf{n}}=\frac{\gamma(\lambda+\mathbf{n} ; \sigma)}{\Gamma(\lambda)} \quad(\lambda \in \mathbb{C}, \mathbf{n} \in \mathbb{N} ; \sigma \geq 0),
$$

and the closely-related incomplete Gamma function $\gamma(s, \sigma)$ is defined by

$$
\gamma(s, \sigma)=\int_{0}^{\sigma} t^{s-1} e^{-t} \mathrm{~d} t \quad(\Re(s)>0, \sigma \geq 0) .
$$

The gamma function and the incomplete gamma function $\gamma(s, \sigma)$ defined by $(11)$ are known to play important and useful roles in the study of the analytic solutions of a variety of problems in diverse areas of science and engineering, see, for example, Srivastava et al. [15], Srivastava and Choi [16, 17], Kumar [18] and others.

\section{REQUiRed INTEGRAL}

We will require the following integral to obtain our main result (see, Brychkov [19, Section (4.1.5), P. 136, Eqn. (37)]):

\section{Lemma 2.1.}

$$
\begin{aligned}
& \int_{0}^{a} x^{s+\frac{1}{2}}(a-x)^{s} \ln \left(b \sqrt[4]{x(a-x)}+\sqrt{1+b^{2} \sqrt{x(a-x)}}\right) \mathrm{d} x \\
&=2^{-2 s-3} \sqrt{\pi} a^{2 s+2} \frac{\Gamma\left(2 s+\frac{5}{2}\right)}{\Gamma(2 s+3)}{ }_{3} F_{2}\left[\begin{array}{c}
\frac{1}{2}, \frac{1}{2}, 2 s+\frac{5}{2} \\
\frac{3}{2}, 2 s+3
\end{array}\right],
\end{aligned}
$$

where $a>0, b \in \mathbb{C}, \Re(s)>-\frac{5}{4},\left|\arg \left(2+a b^{2}\right)\right|<\pi$.

\section{Main Integral}

In this section, we define a general definite integrals involving product of the Alephfunction and generalized incomplete hypergeometric function with general arguments. Here, we note

$$
X=x(a-x),
$$

and

$$
b_{\mathbf{n}}=\frac{\left(e_{1} ; \sigma\right)_{\mathbf{n}}\left(e_{2}\right)_{\mathbf{n}} \cdots\left(e_{\mathbf{p}}\right)_{\mathbf{n}}}{\left(f_{1}\right)_{\mathbf{n}} \cdots\left(f_{\mathbf{q}}\right)_{\mathbf{n}}} .
$$

Then, we have the following formula

\section{Theorem 3.1.}

$$
\begin{aligned}
& \int_{0}^{a} x^{s+\frac{1}{2}}(a-x)^{s} \ln \left(b \sqrt[4]{x(a-x)}+\sqrt{1+b^{2} \sqrt{x(a-x)}}\right) \mathbf{p} \gamma_{\mathbf{q}}\left(y X^{c}\right) \aleph\left(z X^{e}\right) \mathrm{d} x \\
& =2^{-2 s-3} \sqrt{\pi} a^{2 s+2} \sum_{\mathbf{n}, \mathbf{r}=0}^{\infty} \frac{b_{\mathbf{n}} y^{\mathbf{n}}}{\mathbf{n} !}\left(\frac{a}{2}\right)^{2 c \mathbf{n}} \frac{\left(-a b^{2}\right)^{\mathbf{r}}\left(\frac{1}{2}\right)_{\mathbf{r}}\left(\frac{1}{2}\right)_{\mathbf{r}}}{2^{\mathbf{r}}\left(\frac{3}{2}\right)_{\mathbf{r}} \mathbf{r} !} \\
& \times \aleph_{p_{i}+1, q_{i}+1, c_{i} ; r}^{m, n+1}\left[\begin{array}{l|l}
\left(\frac{a}{2}\right)^{2 e} z & \left.\begin{array}{l}
A,\left(a_{j}, A_{j}\right)_{1, n},\left[c_{i}\left(a_{j i}, A_{j i}\right)\right]_{n+1, p_{i} ; r} \\
B,\left(b_{j}, B_{j}\right)_{1, m},\left[c_{i}\left(b_{j i}, B_{j i}\right)\right]_{m+1, q_{i} ; r}
\end{array}\right]
\end{array}\right.
\end{aligned}
$$


where $b_{\mathbf{n}}$ is defined by (14), and

$$
A=\left(-\frac{3}{2}-\mathbf{r}-2(s+\mathbf{n} c) ; 2 e\right) ; \quad B=(-2-\mathbf{r}-2(s+\mathbf{n} c) ; 2 e) .
$$

Also, provided that

(i) $a, e, c>0, b \in \mathbb{C}, \Re(s)>-\frac{5}{4},\left|\arg \left(2+a b^{2}\right)\right|<\pi$.

(ii) $\Re(s+\mathbf{n} c)+e \min _{1 \leq j \leq m} \Re\left(\frac{b_{j}}{B_{j}}\right)>-\frac{5}{4}$.

(iii) $\left|\arg \left(z X^{e}\right)\right|<\frac{1}{2} \pi \varphi_{l} \quad(l=\overline{1, r})$, where $\varphi_{l}$ is defined by $(5)$; and

(iv) $\left(e_{1}, \cdots, e_{\mathbf{p}}, f_{1}, \cdots, f_{\mathbf{q}} \in \mathbb{C}, \mathbf{n} \in \mathbb{N} ; \sigma \geq 0\right)$.

Proof. Expressing extension of the generalized incomplete hypergeometric function $\mathbf{p} \gamma_{\mathbf{q}}($. with the help of the equation (9), and the Aleph-function in Mellin-Barnes integral contour with the help of equations (1) and (2); also changing the order of integration and summation (which is easily seen to be justified due to the absolute convergence of the integral and summation involved in the process. Then, we obtain (say $\mathcal{I}$ )

$$
\begin{aligned}
\mathcal{I}=\sum_{\mathbf{n}=0}^{\infty} \frac{b_{\mathbf{n}} y^{\mathbf{n}}}{\mathbf{n} !} & \int_{0}^{a} x^{s+c \mathbf{n}}(a-x)^{s+c \mathbf{n}+\frac{1}{2}} \ln \left(b \sqrt[4]{x(a-x)}+\sqrt{1+b^{2} \sqrt{x(a-x)}}\right) \\
\times & {\left[\frac{1}{2 \pi \omega} \int_{\mathfrak{L}} \Omega_{p_{i}, q_{i}, c_{i} ; r}^{m, n}(u) z^{-u} x^{-e u}(a-x)^{-e u} \mathrm{~d} u\right] \mathrm{d} x . }
\end{aligned}
$$

Interchanging the order of $x$-integral and $u$-integral (which is permissible under the given conditions), we arrive at

$$
\begin{aligned}
\mathcal{I}=\sum_{\mathbf{n}=0}^{\infty} \frac{b_{\mathbf{n}} y^{\mathbf{n}}}{\mathbf{n} !} \frac{1}{2 \pi \omega} & \int_{\mathfrak{L}} \Omega_{p_{i}, q_{i}, c_{i} ; r}^{m, n}(u) z^{-u}\left[\int_{0}^{a} x^{s+c \mathbf{n}-e u}(a-x)^{s+c \mathbf{n}-e u+\frac{1}{2}}\right. \\
& \left.\times \ln \left(b \sqrt[4]{x(a-x)}+\sqrt{1+b^{2} \sqrt{x(a-x)}}\right) \mathrm{d} u\right] \mathrm{d} x .
\end{aligned}
$$

We evaluate the inner integral with the help of lemma 2.1, then we obtain

$$
\begin{aligned}
& \int_{0}^{a} x^{s+c \mathbf{n}-e u}(a-x)^{s+c \mathbf{n}-e u+\frac{1}{2}} \ln \left(b \sqrt[4]{x(a-x)}+\sqrt{\left.1+b^{2} \sqrt{x(a-x)}\right)} \mathrm{d} x=2^{-2(s+c \mathbf{n}-e u)-3}\right. \\
& \left.\times \sqrt{\pi} a^{2(s+c \mathbf{n}-e u)+2} \frac{\Gamma\left(2(s+c \mathbf{n}-e u)+\frac{5}{2}\right)}{\Gamma(2(s+c \mathbf{n}-e u)+3)}{ }_{3} F_{2}\left[\begin{array}{c}
\frac{1}{2}, \frac{1}{2}, 2(s+c \mathbf{n}-e u)+\frac{5}{2} \\
\frac{3}{2}, 2(s+c \mathbf{n}-e u)+3
\end{array}\right]-\frac{a b^{2}}{2}\right] .
\end{aligned}
$$

Now, we substitute (19) in (18), we get

$$
\begin{aligned}
\mathcal{I}=\sum_{\mathbf{n}=0}^{\infty} & \frac{b_{\mathbf{n}} y^{\mathbf{n}}}{\mathbf{n} !} \frac{1}{2 \pi \omega} \int_{\mathfrak{L}} \Omega_{p_{i}, q_{i}, c_{i} ; r}^{m, n}(u) z^{-u} 2^{-2(s+c \mathbf{n}-e u)-3} \sqrt{\pi} a^{2(s+c \mathbf{n}-e u)+2} \\
& \times \frac{\Gamma\left(2(s+c \mathbf{n}-e u)+\frac{5}{2}\right)}{\Gamma(2(s+c \mathbf{n}-e u)+3)}{ }_{3} F_{2}\left[\begin{array}{c}
\frac{1}{2}, \frac{1}{2}, 2(s+c \mathbf{n}-e u)+\frac{5}{2} \\
\frac{3}{2}, 2(s+c \mathbf{n}-e u)+3
\end{array} \mid-\frac{a b^{2}}{2}\right] .
\end{aligned}
$$


We express the hypergeometric function ${ }_{3} F_{2}($.$) in series, we have$

$$
\begin{aligned}
\mathcal{I} & =\sum_{\mathbf{n}=0}^{\infty} \frac{b_{\mathbf{n}} y^{\mathbf{n}}}{\mathbf{n} !} \frac{1}{2 \pi \omega} \int_{\mathfrak{L}} \Omega_{p_{i}, q_{i}, c_{i} ; r}^{m, n}(u) z^{-u} 2^{-2(s+c \mathbf{n}-e u)-3} \sqrt{\pi} a^{2(s+c \mathbf{n}-e u)+2} \\
& \times \sum_{\mathbf{r}=0}^{\infty} \frac{\left(\frac{1}{2}\right)_{\mathbf{r}}\left(\frac{1}{2}\right)_{\mathbf{r}} \Gamma\left(2(s+c \mathbf{n}-e u)+\mathbf{r}+\frac{5}{2}\right)}{\left(\frac{3}{2}\right)_{\mathbf{r}} \Gamma(2(s+c \mathbf{n}-e u)+3+\mathbf{r}) \mathbf{r} !}\left(-\frac{a b^{2}}{2}\right)^{\mathbf{r}} \mathrm{d} u \\
& =2^{-2 s-3} \sqrt{\pi} a^{2 s+2} \sum_{\mathbf{n}=0}^{\infty} \frac{b_{\mathbf{n}} y^{\mathbf{n}}}{\mathbf{n} !} \frac{1}{2 \pi \omega} \int_{\mathfrak{L}} \Omega_{p_{i}, q_{i}, c_{i} ; r}^{m, n}(u) z^{-u} 2^{-2(c \mathbf{n}-e u)} \sqrt{\pi} a^{2(c \mathbf{n}-e u)} \\
& \times \sum_{\mathbf{r}=0}^{\infty} \frac{\left(\frac{1}{2}\right)_{\mathbf{r}}\left(\frac{1}{2}\right)_{\mathbf{r}} \Gamma\left(2(s+c \mathbf{n}-e u)+\mathbf{r}+\frac{5}{2}\right)}{\left(\frac{3}{2}\right)_{\mathbf{r}} \Gamma(2(s+c \mathbf{n}-e u)+3+\mathbf{r}) \mathbf{r} !}\left(-\frac{a b^{2}}{2}\right)^{\mathbf{r}} \mathrm{d} u
\end{aligned}
$$

Now, we change the order of integration and summation (which is easily seen to be justified due to the absolute convergence of the $u$-integral and the $\mathbf{n}$-series involved in the process), then we obtain

$$
\begin{aligned}
& \mathcal{I}=2^{-2 s-3} \sqrt{\pi} a^{2 s+2} \sum_{\mathbf{n}, \mathbf{r}=0}^{\infty} \frac{b_{\mathbf{n}} y^{\mathbf{n}}}{\mathbf{n} !} \frac{\left(-a b^{2}\right)^{\mathbf{r}}\left(\frac{1}{2}\right)_{\mathbf{r}}\left(\frac{1}{2}\right)_{\mathbf{r}}}{2^{\mathbf{r}}\left(\frac{3}{2}\right)_{\mathbf{r}} \mathbf{r} !}\left(\frac{a}{2}\right)^{2 c \mathbf{n}} \\
& \times \frac{1}{2 \pi \omega} \int_{\mathfrak{L}} \Omega_{p_{i}, q_{i}, c_{i} ; r}^{m, n}(u) z^{-u} 2^{2 e u} a^{-2 e u} \frac{\Gamma\left(2(s+c \mathbf{n}-e u)+\mathbf{r}+\frac{5}{2}\right)}{\Gamma(2(s+c \mathbf{n}-e u)+3+\mathbf{r})} \mathrm{d} u .
\end{aligned}
$$

Finally, interpreting the result (22) thus obtained with the Mellin-barnes integral contour, we arrive at the desired formula (15).

\section{Particular Cases}

In this section, we shall obtain two corollaries concerning $I$ - and $H$-function.

Corollary 4.1. Take relation (7) account in (15), then we obtain integral involving Ifunction [11] and the generalized incomplete hypergeometric function, defined by

$$
\begin{aligned}
& \int_{0}^{a} x^{s+\frac{1}{2}}(a-x)^{s} \ln \left(b \sqrt[4]{x(a-x)}+\sqrt{1+b^{2} \sqrt{x(a-x)}}\right) \mathbf{p} \gamma_{\mathbf{q}}\left(y X^{c}\right) I\left(z X^{e}\right) \mathrm{d} x \\
& =2^{-2 s-3} \sqrt{\pi} a^{2 s+2} \sum_{\mathbf{n}, \mathbf{r}=0}^{\infty} \frac{b_{\mathbf{n}} y^{\mathbf{n}}}{\mathbf{n} !}\left(\frac{a}{2}\right)^{2 c \mathbf{n}} \frac{\left(-a b^{2}\right)^{\mathbf{r}}\left(\frac{1}{2}\right)_{\mathbf{r}}\left(\frac{1}{2}\right)_{\mathbf{r}}}{2^{\mathbf{r}}\left(\frac{3}{2}\right)_{\mathbf{r}} \mathbf{r} !}
\end{aligned}
$$

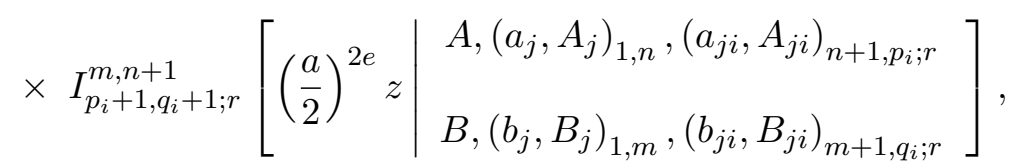

under the same notations and conditions (i), (ii) and (iv) that stated in (15); also satisfy

$$
\left|\arg \left(z X^{e}\right)\right|<\frac{1}{2} \pi \varphi_{l}^{\prime} \quad(l=\overline{1, r}) \text {, where } \varphi_{l}^{\prime}=\sum_{j=1}^{n} A_{j}-\sum_{j=n+1}^{p_{i}} A_{j i}+\sum_{j=1}^{m} B_{j}-\sum_{j=m+1}^{q_{i}} B_{j i} .
$$

Next, we use the relation (8) in the above corollary, then the $I$-function of one variable reduces to the Fox's $H$-function [12] (see also, $[13,14,5]$ ), then we arrive at the following corollary concerning the integral involving $H$-function and the generalized incomplete hypergeometric function, given as 


\section{Corollary 4.2.}

$$
\begin{aligned}
& \int_{0}^{a} x^{s+\frac{1}{2}}(a-x)^{s} \ln \left(b \sqrt[4]{x(a-x)}+\sqrt{1+b^{2} \sqrt{x(a-x)}}\right) \mathbf{p} \gamma_{\mathbf{q}}\left(y X^{c}\right) H\left(z X^{e}\right) \mathrm{d} x \\
& =2^{-2 s-3} \sqrt{\pi} a^{2 s+2} \sum_{\mathbf{n}, \mathbf{r}=0}^{\infty} \frac{b_{\mathbf{n}} y^{\mathbf{n}}}{\mathbf{n} !}\left(\frac{a}{2}\right)^{2 c \mathbf{n}} \frac{\left(-a b^{2}\right)^{\mathbf{r}}\left(\frac{1}{2}\right)_{\mathbf{r}}\left(\frac{1}{2}\right)_{\mathbf{r}}}{2^{\mathbf{r}}\left(\frac{3}{2}\right)_{\mathbf{r}} \mathbf{r} !} \\
& \times H_{p+1, q+1 ; r}^{m, n+1}\left[\begin{array}{l|l}
\left(\frac{a}{2}\right)^{2 e} z & \begin{array}{l}
A,\left(a_{j}, A_{j}\right)_{1, p} \\
B,\left(b_{j}, B_{j}\right)_{1, q}
\end{array}
\end{array}\right],
\end{aligned}
$$

under the same notations and conditions (i), (ii) and (iv) that stated in (15); and satisfy

$$
\left|\arg \left(z X^{e}\right)\right|<\frac{1}{2} \pi \varphi \text {, where } \varphi=\sum_{j=1}^{n} A_{j}-\sum_{j=n+1}^{p} A_{j}+\sum_{j=1}^{m} B_{j}-\sum_{j=m+1}^{q} B_{j} .
$$

Remark 4.1. By using the similar methods, we can obtain the analog relations with the $I$-function of one variable defined by Rathie [20], the H-function defined by Buschman and Srivastava [21], Inayat [22], the A-function of one variable defined by Gautam et al.[23].

\section{Concluding Remarks}

We conclude our present study by remarking that the several further consequences of our results can easily be derived by using some known and new relationships between Alephfunctions, which is an elegant unification of various special functions such as Fox $H$ - and I-function (see $[11,12,13,14,24]$ ), after some suitable parametric replacements. Secondly, specializing the parameters of the generalized incomplete hypergeometric function $\mathbf{p} \gamma_{\mathbf{q}}($.$) ,$ we can obtain others special functions. The integral obtained in this paper is of the general nature, we can get known and new integrals by specializing the different parameters.

\section{REFERENCES}

[1] Südland, N., Baumann, B., Nonnenmacher, T.F., (1998), Open problem: Who knows about the Alephfunction?, Fract. Calc. Appl. Anal., 1(4), pp. 401-402.

[2] Südland, N., Baumann, B., Nonnenmacher, T.F., (2001), Fractional drift-less Fokker-Planck equation with power law diffusion coefficients, (in V.G. Gangha, E.W. Mayr, W.G. Vorozhtsov (Eds.)), Computer Algebra in Scientific Computing (CASC Konstanz 2001), Springer, Berlin, pp. 513-525.

[3] Ayant, F. Y. and Kumar, D., (2016), Certain finite double integrals involving the hypergeometric function and Aleph-function, International Journal of Mathematics Trends and Technology, 35 (1), pp 49-55.

[4] Choi, J. and Kumar, D., (2014), Certain unified fractional integrals and derivatives for a product of Aleph function and a general class of multivariable polynomials, Journal of Inequalities and Applications, 2014, pp. 1-15.

[5] Ram, J. and Kumar, D., (2011), Generalized fractional integration of the $\aleph$-function, J. Raj. Acad. Phy. Sci, 10(4), pp. 373-382.

[6] Saxena, R. K. and Kumar, D., (2015), Generalized fractional calculus of the Aleph-function involving a general class of polynomials, Acta Mathematica Scientia, 35(5), pp. 1095-1110.

[7] Saxena, R. K. and Pogány, T.K., (2011), On fractional integration formulae for Aleph functions, Appl. Math. Comput., 218, pp. 985-990.

[8] Saxena, R. K., Ram, J., Kumar, D., (2013), Generalized fractional integral of the product of two Aleph-functions, Applications and Applied Mathematics, 8(2), pp. 631-646.

[9] Saxena, R. K., Ram, J., Kumar, D., (2018), Finite Integral Formulas Involving Aleph Function, Boletim da Sociedade Paranaense de Matematica, 36, (1), pp. 177-193.

[10] N. Südland, J. Volkmann and D. Kumar, Applications to give an analytical solution to the Black Scholes equation, Integral Transforms Spec. Funct., 30(3) (2019), 205-230. 
[11] Saxena, V. P., (2001), Formal solution of certain new pair of dual integral equations involving $H$ function, Proc. Nat. Acad. Sci. India Sect., A51, pp. 366-375.

[12] Fox, C. (1961), The $G$ and $H$-functions as symmetrical Fourier kernels, Trans. Amer. Math. Soc., 98, pp. 395-429.

[13] Mathai, A. M. and Saxena, R. K., (1978), The $H$-function with Applications in Statistics and Other Disciplines, Halsted Press (John Wiley \& Sons), New York-London-Sydney-Toronto.

[14] Mathai, A. M, Saxena, R. K., Haubold, H. J., (2010), The $H$-function: Theory and Applications, Springer, New York.

[15] Srivastava, H. M., Chaudhry, M. A., Agarwal, R. P., (2012), The incomplete Pochhammer symbols and their in applications to hypergeometric and related functions, Integral Transforms ans Special Functions, 23, pp. 659-683.

[16] Srivastava, H. M. and Choi, J., (2001), Series associated with the Zeta and related functions, Kluwer Academic Publishers, Dordrecht-Boston-London.

[17] Srivastava, H. M. and Choi, J., (2012), Zeta and $q$-Zeta functions and associated series and integrals, Elsevier Science Publishers, Amsterdam-London-New York.

[18] Kumar, D. (2016), Certain integrals of generalized hypergeometric and confluent hypergeometric functions, Sigmae, 5(2), pp. 8-18.

[19] Brychkov, Y. A., (2008), Handbook of Special Functions: Derivatives, Integrals, Series and Other Formulas, Chapman and Hall/CRC Press, Taylor and Francis Group, New York.

[20] Rathie, A. K., (1997), A new generalization of generalized hypergeometric functions, Le Matematiche, 52(2), pp. 297-310.

[21] Buschman, R. G. and Srivastava, H. M., (1990), The $H$-function associated with a certain class Feynman-integrals, J. Phys. Math. Gen., 23, pp. 4707-4710.

[22] Inayat, A. A. H., (1987), New Properties of Hypergeometric series derivable from Feynmann integrals II, A generalization of the $H$-function, J. Phys. A. Math. Gen., 20, pp. 4119-4128.

[23] Gautam, B. P., Asgar, A. S., Goyal, A.N., (1980), The A-function, Revista Mathematica, Tucuman.

[24] Kilbas, A. A., Srivastava, H. M., Trujillo, J. J., (2006), Theory and Applications of Fractional Differential Equations, North-Holland Mathematical Studies, Vol. 204, Elsevier (North Holland) Science Publishers, Amsterdam-London-New York.

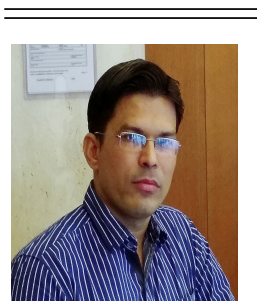

Dinesh Kumar is working as an assistant professor of mathematics in the Department of Applied Sciences in Agriculture University-Jodhpur, Rajasthan, India. He has published 68 research papers in National and International peer reviewed journals so far, and publish one reference book with international publisher. His research interest includes Fractional Calculus, Special Functions, Integral Transforms, $q$-Fractional Calculus, Mathematical Physics, Differential Equation, Basic Hypergeometric functions, and Kinetic and Reaction-Diffusion Equations.

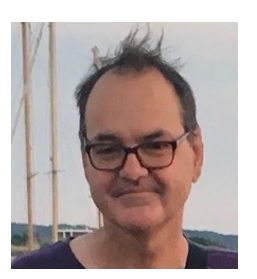

Frédéric Ayant is a lecturer in high school and have experienced to work at Collége Jean L'herminier, Allée des Nymphéas, 83500 La Seyne-sur-Mer, France. His research interested are fractional calculus, integral transforms, mathematical physics, mathematical modeling, special function and many more. Recently, he is working on fractional calculus operators and their applications in related fields.

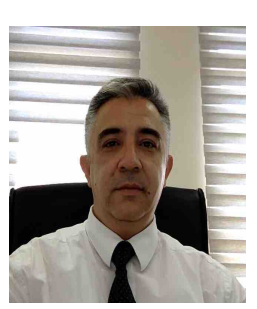

Faruk Uçar has been working in the Department of Mathematics, Marmara University since 1993. He received his Ph.D. degree in Univalent functions in 2004 from Marmara University, Istanbul, Turkey. His primary areas of research are univalent functions, integral transforms and $q$-analysis. 